\title{
Retraction
}

\section{Retracted: Dual-Band Microstrip Patch Antenna Miniaturization Using Metamaterial}

\author{
Journal of Engineering \\ Received 7 July 2013; Accepted 7 July 2013 \\ Copyright (C) 2013 Journal of Engineering. This is an open access article distributed under the Creative Commons Attribution \\ License, which permits unrestricted use, distribution, and reproduction in any medium, provided the original work is properly \\ cited.
}

This article has been retracted as it is found to contain a substantial amount of materials, without referencing, from the article "Microstrip Patch Antenna Miniaturization using Artificial Substrate," by Sanket S. Patel and Sanjeev Gupta which was published in the Fifth Annual Conference Antenna Test and Measurement Society (ATMS) India - 2012 [1].

\section{References}

[1] I. Singh, V. S. Tripathi, and S. Tiwari, "Dual-band microstrip patch antenna miniaturization using metamaterial," Journal of Engineering, vol. 2013, Article ID 928078, 5 pages, 2013. 


\author{
Indrasen Singh, ${ }^{1}$ Vijay Shanker Tripathi, ${ }^{1}$ and Sudarshan Tiwari ${ }^{2}$ \\ ${ }^{1}$ Department of Electronics and Communication Engineering, Motilal Nehru National Institute of Technology, \\ Allahabad 211004, India \\ ${ }^{2}$ Department of Electronics and Communication Engineering, National Institute of Technology Raipur, Raipur, \\ Chhattisgarh 492010, India
}

Correspondence should be addressed to Indrasen Singh; erindrasen@gmail.com

Received 6 September 2012; Revised 22 March 2013; Accepted 22 March 2013

Academic Editor: Alfio D. Grasso

Copyright (C 2013 Indrasen Singh et al. This is an open access article distributed under the Creative Commons Attribution License, which permits unrestricted use, distribution, and reproduction in any medium, provided the original work is properly cited.

A dual-band microstrip patch antenna is designed and analyzed using metamaterial artificial substrate. Metamaterial based substrate is designed using Square Split Ring Resonator (SSRR) and Wire Strip. The antenna is tuned to work at two resonating frequencies in the frequency range from $1 \mathrm{GHz}$ to $4 \mathrm{GHz}$ depending on the geometric specifications of SSRR, strip line, radiating patch, and feed location point. Proposed antenna provides good return loss behavior at both resonating frequencies. The obtained VSWR at both resonating frequencies is very much near to 1 . Proposed antenna covers applications in mobile communication and Wi-MAX. Proposed patch antenna is compared with the conventional patch antenna, which shows the significant miniaturization as compared to conventional patch antenna.

\section{Introduction}

The demand for small, compact, low-cost antennas has increased tremendously over the past years. Various types of microstrip patch antenna and its applications are discussed [1]. Due to the need for reduced antenna size in both military and commercial spheres, a new approach of miniaturization using metamaterial is proposed in this paper. Metamaterials are a new class of ordered composites that exhibit exceptional properties not readily observed in nature. These properties arise from qualitatively new response functions that are not observed in the constituent materials and result from the inclusion of artificially fabricated, extrinsic, low dimensional inhomogeneities. Such material exhibits permittivity and permeability both negative, and hence they are known as the double negative (DNG) materials [2-4]. And so they have Negative Refractive Index (NRI), and hence they are also called as the Negative Refractive Index materials or Left-handed material LHM (as they follow left-hand rule)
[5-7]. Because of their unusual properties, they offer some interesting changes in radiation characteristics of an antenna. The idea of MTMs has been quickly adopted in research, due to rapidly developing nanofabrication and subwavelength imaging techniques [8]. The square split ring resonator and its equivalent electrical circuit are given in Figure 1.

\section{Antenna Design}

Proposed patch antenna is a design using artificial substrate made up of metamaterial using SRR and wire strip. Here square-shaped split ring resonator and a wire strip are used to make metamaterial design. Wire strip ensures negative permittivity and SRR ensures the negative permeability [10]. When both structures are combined together, then they simultaneously offer negative permittivity, and negative permeability and hence it becomes double negative metamaterial. The geometry of proposed patch antenna with parameters is shown in Figure 2. The antenna is mounted on an 


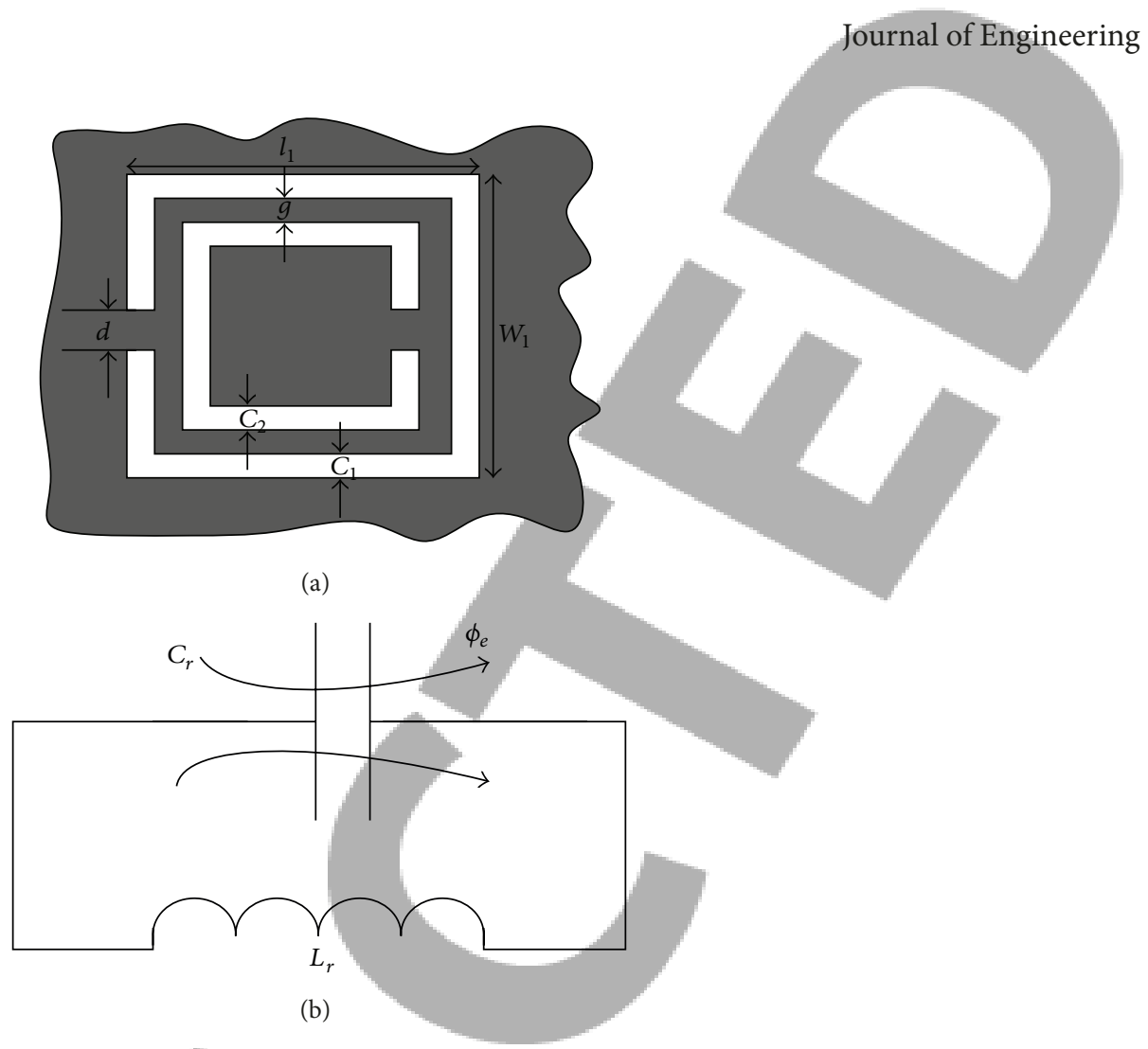

FIGURE 1: (a) Topology and (b) its equivalent circuit model of the SSRR [9]. Gray zone represents the metallization.

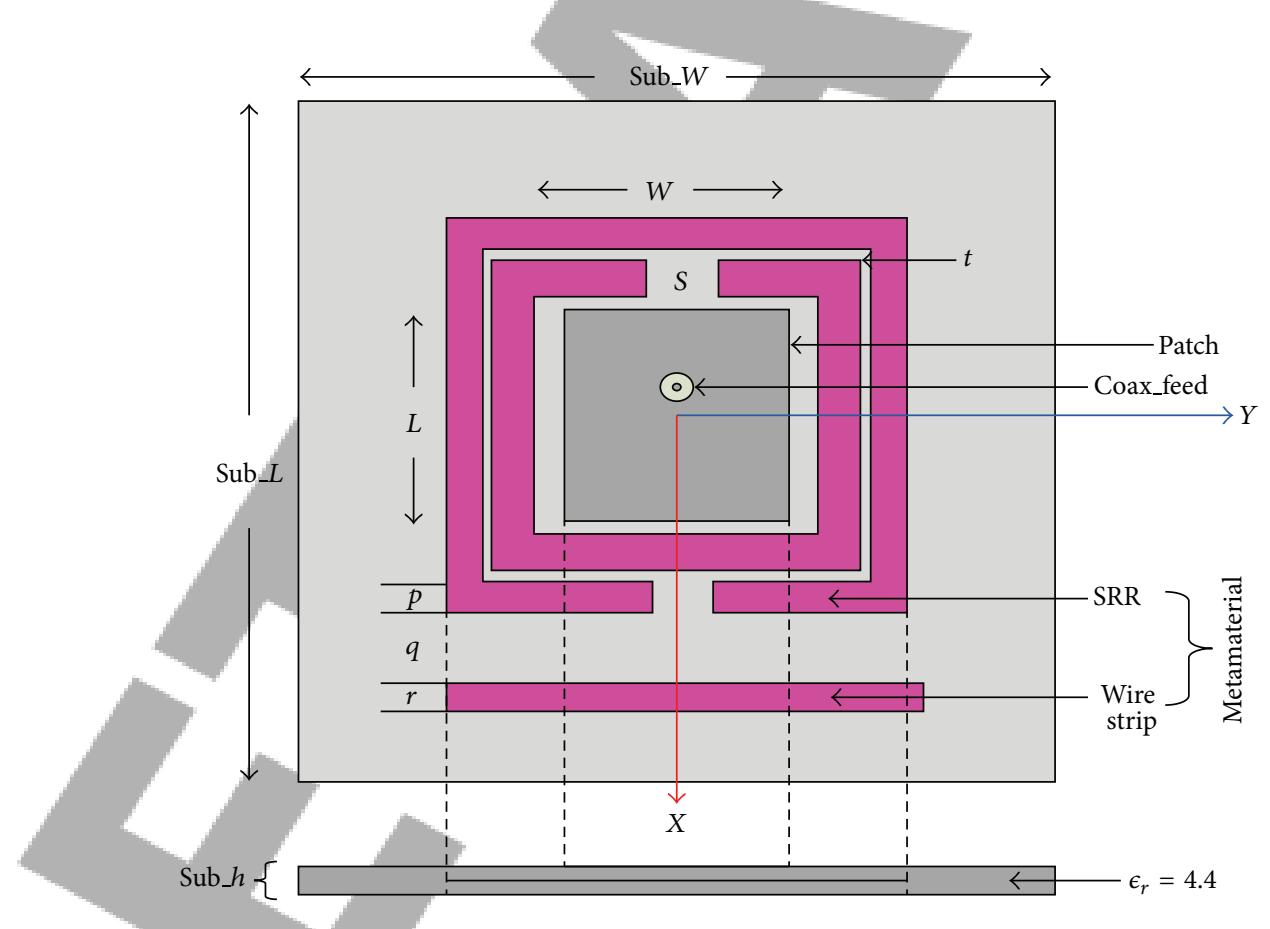

FIGURE 2: Square SRR based metamaterial inserted antenna layout. Parameters are sub_W $=90 \mathrm{~mm}$, sub $L=100 \mathrm{~mm}, W=30 \mathrm{~mm}, L=$ $40 \mathrm{~mm}$, sub $h=3.2 \mathrm{~mm}, p=5 \mathrm{~mm}, q=10 \mathrm{~mm}, r=5 \mathrm{~mm}, s=5 \mathrm{~mm}$, and $t=1 \mathrm{~mm}$.

FR4_epoxy substrate and fed by a coaxial transmission line. Patch and ground plane are made of copper having relative permittivity as $\epsilon_{r}=1$. The substrate material FR4_epoxy has relative permittivity as $\epsilon_{r}=4.4$ and dielectric loss tangent $\tan \delta=0.025$. SRR and wire strip structure is made of copper and inserted in between the substrate as shown in Figure 2 . Copper thickness is $0.03 \mathrm{~mm}$. Excitation to patch is given by coaxial wave port. Convergence was tested for each case 


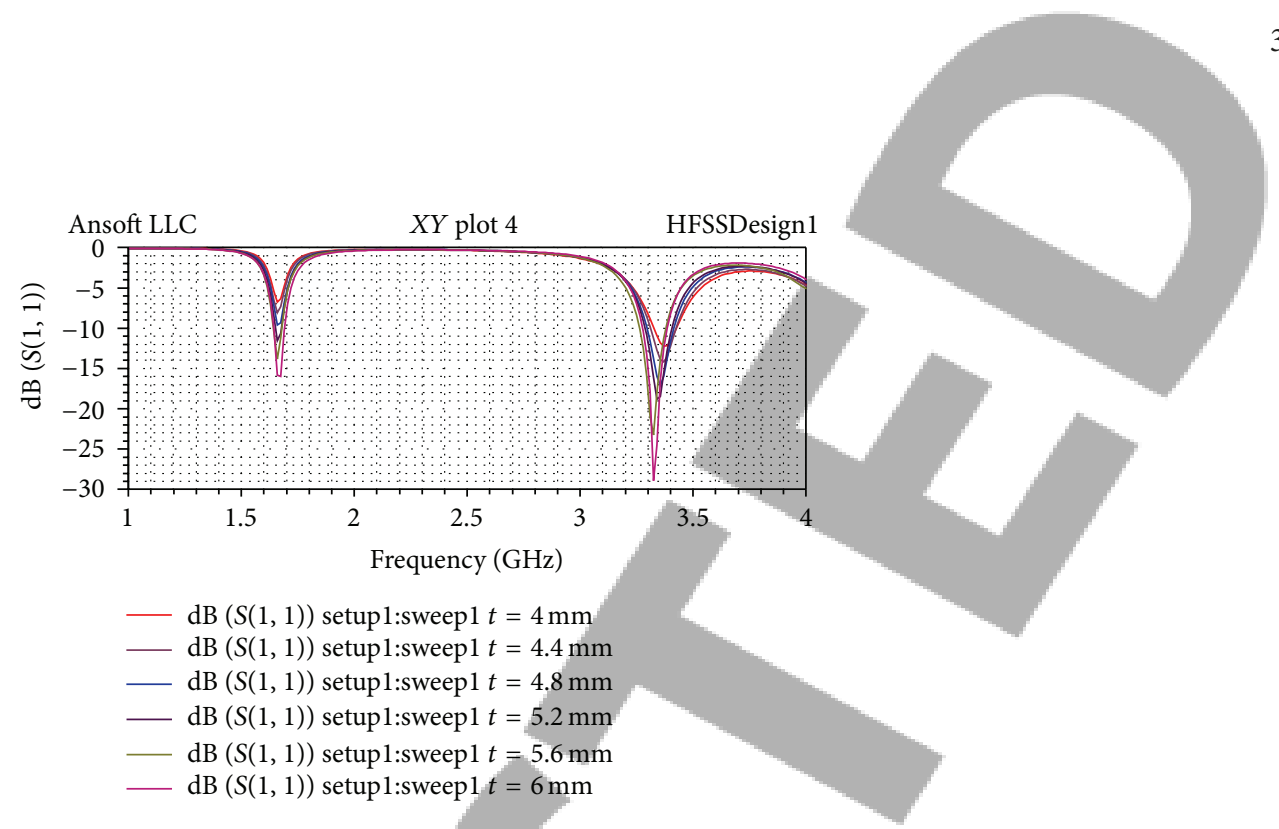

FIGURE 3: Optimize return loss characteristics of dual-band patch antenna.

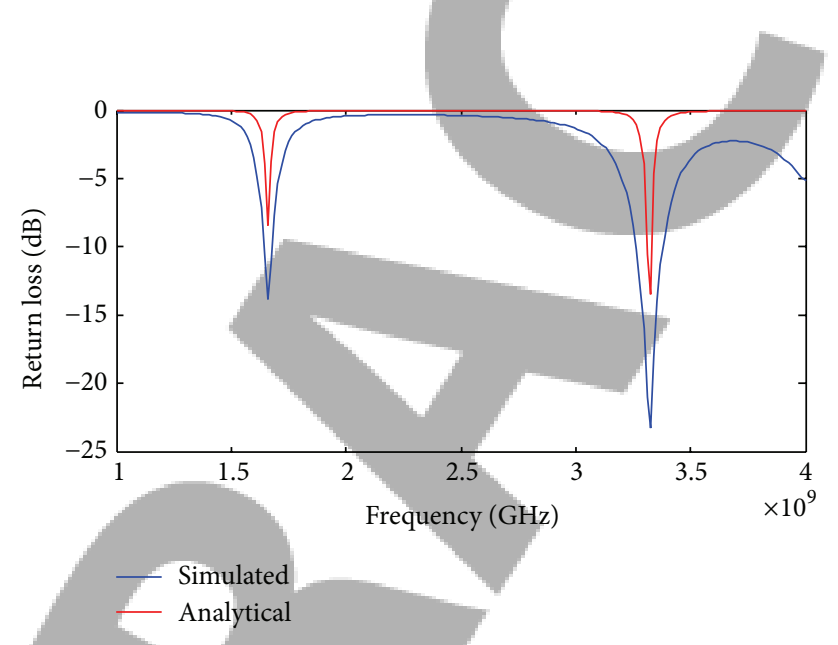

FIGURE 4: Return loss characteristics of dual-band patch antenna.

separately in terms of evaluating $S_{11}(\mathrm{~dB})$ at a single frequency for a number of times. Once convergence was obtained, simulations were conducted in order to obtain swept frequency response extending from 1 to $4 \mathrm{GHz}$. The swept response gave us the $S_{11}$, which was used to calculate the VSWR. Here the simulation is performed using Ansoft's High Frequency Structure Simulator (HFSS) software package [11]. HFSS provides $E$-fields and $H$-fields, currents, $S$-parameters, and near and far radiated field results. It integrates simulation, visualization, solid modeling, and automation. Ansoft HFSS employs the Finite Element Method (FEM) for EM simulation by developing/implementing technologies such as tangential vector finite elements and adaptive meshing (Figure 5).

\section{Antenna Miniaturization}

Now we will do the comparative analysis of the proposed metamaterial antenna with the conventional antenna design.
Both antenna configurations are shown in Figures 7 and 8. In both antennas, the material used for the substrate is the same FR4_epoxy having dielectric constant as $\epsilon_{r}=4.4$. Patch and other conductive elements in both antennas are made of copper with the copper thickness of $0.03 \mathrm{~mm}$. Return loss characteristics for both conventional antenna and proposed antenna are shown in Figure 9. First metamaterial antenna is designed and then conventional patch antenna is designed considering the same materials as discussed. Conventional patch antenna is tuned to have the same kind of response as that of the proposed metamaterial based antenna. From Figure 9, it is clear that both antennas have the same operating frequency and they offer almost the same bandwidth. After thorough analysis, it is found that proposed metamaterial based antenna requires smaller size compared to the conventional simple rectangular patch antenna. The reduction in patch size is about $84 \%$ which is shown in Table 1. So antenna miniaturization has been successfully carried out using metamaterial concept. 


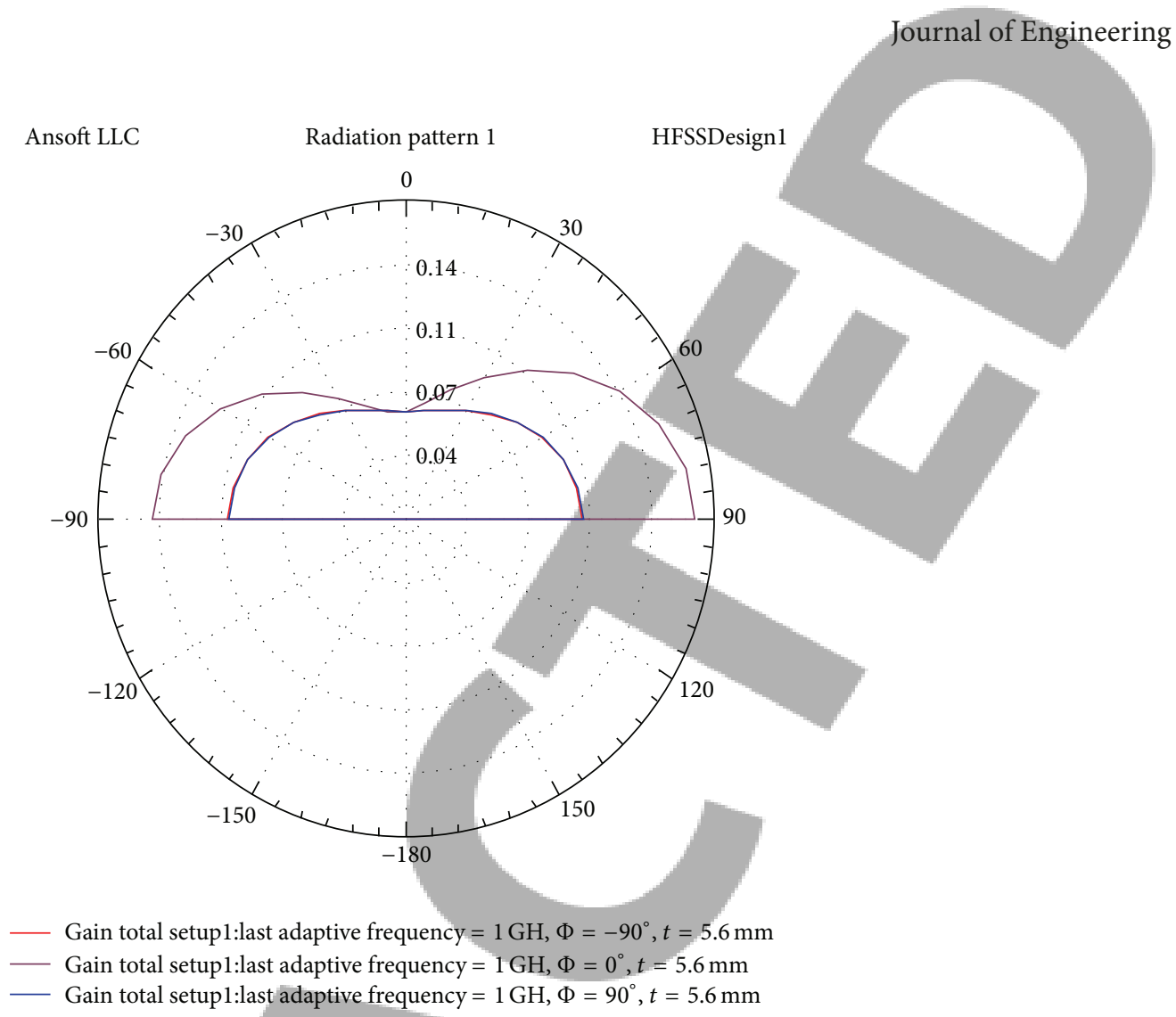

FIGURE 5: Radiation pattern (gain) of proposed patch antenna.

TABLE 1: Analysis of antenna miniaturization carried out using metamaterial design.

\begin{tabular}{lccc}
\hline Parameter & Conventional antenna & Proposed metamaterial based antenna & Reduction in percentage \\
\hline Active patch area & $92 \mathrm{~mm} \times 82 \mathrm{~mm}=7544 \mathrm{~mm}^{2}$ & $40 \mathrm{~mm} \times 30 \mathrm{~mm}=1200 \mathrm{~mm}^{2}$ & $84.093 \%$ \\
Volume of antenna & $152 \mathrm{~mm} \times 142 \mathrm{~mm} \times 3.2 \mathrm{~mm}=69068.8 \mathrm{~mm}^{3}$ & $100 \mathrm{~mm} \times 90 \mathrm{~mm} \times 3.2 \mathrm{~mm}=28800 \mathrm{~mm}^{3}$ & $58.302 \%$ \\
\hline
\end{tabular}

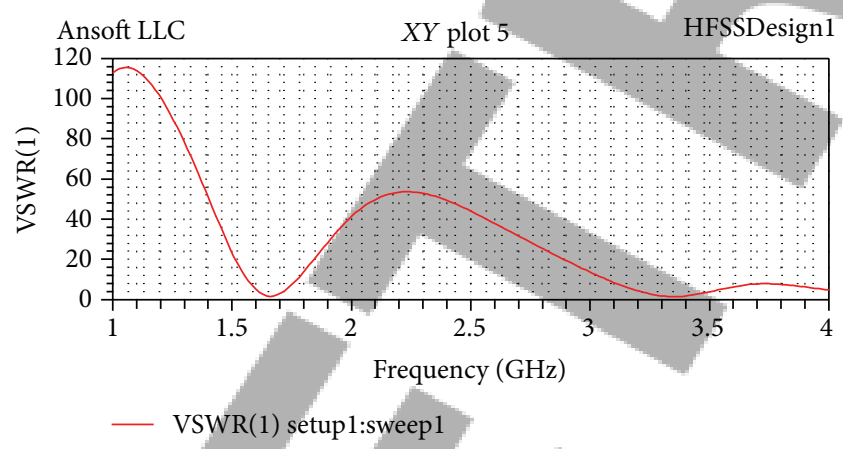

FIGURE 6: VSWR v/s frequency plot for dual-band proposed patch antenna.

\section{Conclusion}

A patch antenna miniaturization technique using metamaterial having square split ring resonator and wire strip is proposed and compared with conventional patch antenna. The SRR and strip wire loading reduces the physical size of the patch significantly as compared to the conventional one. Return loss characteristics are observed for the proposed

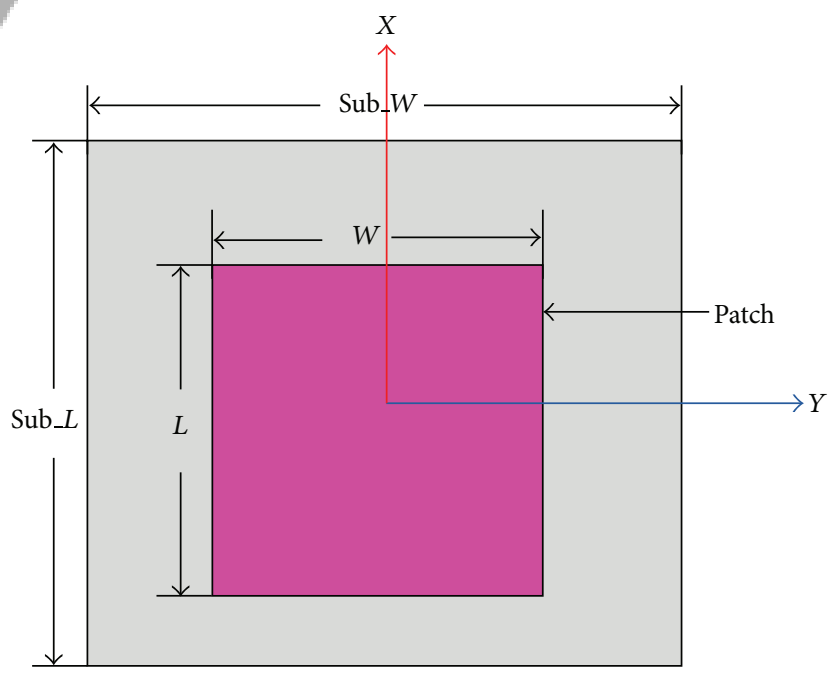

FIGURE 7: Conventional microstrip antenna.

antenna configuration by and depicted in Figures 3 and 4 . It is shown as both simulated and analytical results very near 


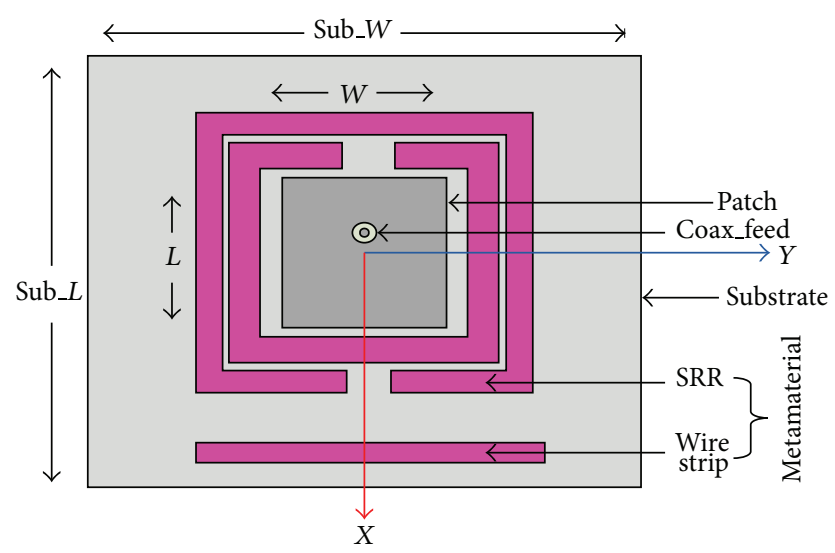

FIGURE 8: Metamaterial based microstrip patch antenna.

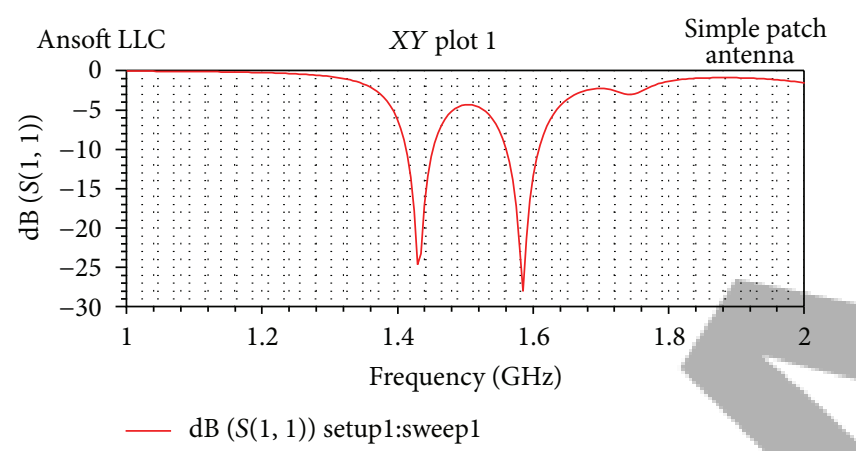

(a)

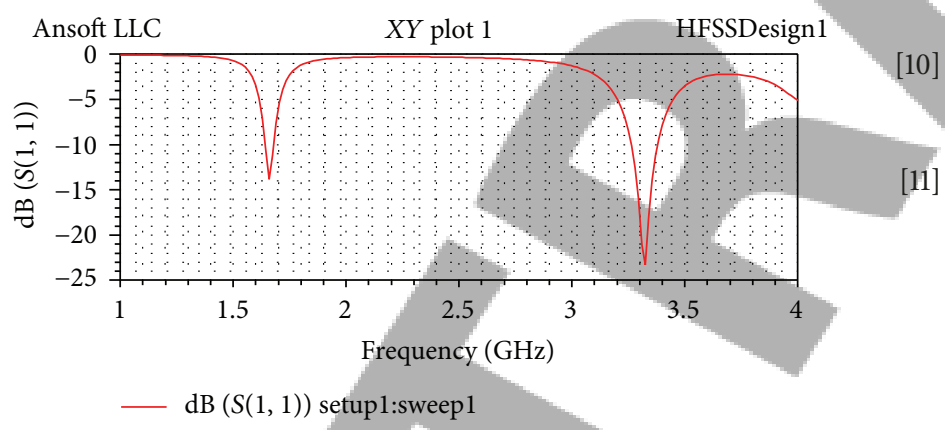

(b)

FIGURE 9: Return loss plots for conventional simple rectangular patch antenna (a) and proposed antenna design (b).

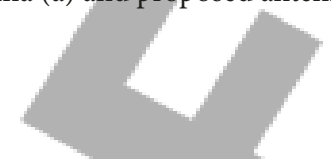

to each other. From Figure 9, it is concluded that antenna gives dual-band of operation in between $1 \mathrm{GHz}$ to $4 \mathrm{GHz}$ range of frequency. From Figure 4: percentage bandwidth observed at $-10 \mathrm{~dB}$ return loss for the first band is $2.40 \%$ and for second band is $2.50 \%$. Corresponding VSWR plot for the same antenna is shown in Figure 6. VSWR values obtained at first and second resonating frequencies are 1.3572 and 1.0942 which is very much nearer to ideal value 1 for both frequencies. The proposed antenna is a good candidate for a mobile communication and Wi-Max applications.

\section{References}

[1] I. Singh and V. S. Tripathi, "Microstrip patch antenna and its applications: a survey," International Journal of Computer Technology and Applications, no. 5, pp. 1595-1599, 2011.

[2] V. Veselago, "The electrodynamics of substances with simultaneously negative values of $\varepsilon$ and $\mu$," Soviet Physics Uspekhi, vol. 10, no. 4, pp. 509-514, 1968.

[3] J. B. Pendry, A. J. Holden, D. J. Robbins, and W. J. Stewart, "Low frequency plasmons in thin-wire structures," Journal of Physics: Condensed Matter, vol. 10, pp. 4785-4809, 1998.

[4] R. W. Ziolkowski, "Design, fabrication, and testing of double negative metamaterials," IEEE Transactions on Antennas and Propagation, vol. 51, no. 7, pp. 1516-1529, 2003.

[5] D. R. Smith, W. J. Padilla, D. C. Vier, S. C. Nemat Nasser, and S. Schultz, "Composite medium with simultaneously negative permeability and permittivity," Physical Review Letters, vol. 84, no. 18, pp. 4184-4187, 2000.

[6] R. A. Shelby, D. R. Smith, and S. Schultz, "Experimental verification of a negative index of refraction," Science, vol. 292, no. 5514, pp. 77-79, 2001.

[7] R. W. Ziolkowski and E. Heyman, "Wave propagation in media having negative permittivity and permeability," Physical Review E, vol. 64, no. 5, pp. 1-15, 2001.

[8] Y. Dong and T. Itoh, "Metamaterial-inspired broadband mushroom antenna," in IEEE Antennas and Propagation Society International Symposium (APSURSI '10), pp. 1-4, Toronto, Canada, 2010.

[9] J. D. Baena, J. Bonache, F. Martín et al., "Equivalent-circuit models for split-ring resonators and complementary splitring resonators coupled to planar transmission lines," IEEE Transactions on Microwave Theory and Techniques, vol. 53, no. 4, pp. 1451-1460, 2005.

[10] R. W. Ziolkowski and A. Erentok, "Metamaterial-based efficient electrically small antennas," IEEE Transactions on Antennas and Propagation, vol. 54, no. 7, pp. 2113-2130, 2006.

[11] HFSS ver. 12 ANSYS, http://www.ansys.com/ANSYS+HFSS. 